Congreso Internacional de Gestión Educativa, 06 y 07 de noviembre de 2020.

Pontificia Universidad Católica del Perú

\title{
Gestión educativa en tiempos de pandemia de la Región Puno, 2020
}

Benavente Llerena, Mario Fernando

Director Regional de Educación Puno

maferbl167@ hotmail.com

https://orcid.org/0000-0001-7042-4296

\section{Resumen}

El propósito de nuestra gestión está centrado en el liderazgo directivo de comunicación, participación y la búsqueda de alianzas estratégicas con otras entidades del Estado; la pandemia nos ha ilustrado la realidad del sistema educativo en la región, la brecha digital, el uso de las herramientas tecnológicas, no estaban previstas; al inicio, ha alcanzado un porcentaje alto, donde los estudiantes, simplemente se quedaron sin atención en el servicio educativo, en los lugares de difícil acceso, hasta el momento no se cuenta con recursos, con el poco recurso, la entidad hace denodados esfuerzos a fin de contar y asistir a ellos. Hemos movilizado la capacidad de liderazgo, promoviendo la emulación, para firmar convenios con autoridades ediles, éstos por la función y buena voluntad, apoyaron con la implementación de la estrategia "aprendo en casa", que se concretó con la instalación de antenas, la compra de internet satelital para ayudar a los estudiantes y éstas recibir y conectarse en el aprendizaje virtual. El avance del nivel de aprendizaje es abismal e inequitativo, no se puede hacer una comparación, con un estudiante que tiene las condiciones mínimas de aprendizaje, con uno que tiene que caminar kilómetros a fin de captar una señal y conformarse con un dial de sonido a veces defectuoso; el Estado debe estar presente, ellos cuentan para mí patria, y Puno es maravilloso.

Palabras clave: aprendizaje virtual, brecha digital, gestión, liderazgo directivo, emulación, internet.

\begin{abstract}
The purpose of our management is centered on the directive leadership of communication, participation and the search for strategic alliances with other State entities; The pandemic has illustrated us the reality of the educational system in the region, the digital divide, the use of technological tools, were not foreseen; At the beginning, it has reached a high percentage, where students simply were left without attention in the educational service, in places of difficult access, until now there are no resources, with little resources, the entities make strenuous efforts to order to count and attend them. We have mobilized our leadership capacity to sign agreements with municipal authorities, these by function and good will, supported with the implementation of the strategy "I learn at home", which was specified with the installation of antennas, the purchase of satellite internet to help students and they receive and connect in virtual learning. The advancement of the level of learning is abysmal and inequitable, a comparison cannot be made, with a student who has the minimum learning conditions, with one who has to walk kilometers in order to capture a signal and settle for a sound dial sometimes defective; the State must be present, they count for my country, and Puno is wonderful.
\end{abstract}


Congreso Internacional de Gestión Educativa, 06 y 07 de noviembre de 2020.

Pontificia Universidad Católica del Perú

Keywords: virtual learning, digital divide, management, executive leadership, emulation, internet

\section{Introducción}

Nuestra institución se ha encargado de mediar la acción pedagógica, con asistencia técnica en el uso de las herramientas digitales de nuestros docentes y colaboradores, hemos logrado que docentes y estudiantes sopesen la emergencia de la educación, a través de una articulación de acciones estratégicas; se ha atendido a los estudiantes que emprendían largas caminatas para acceder a la conexión, a ellos en especial, hemos atendido en dar soporte técnico y socioemocional a través de sus docentes y la comunidad de manera oportuna; es verdad, con el poco recurso con que contamos y del Estado; pero en la práctica, la estrategia colaborativa ha hecho visible que no se ha abandonado, estamos presentes por todas las dificultades económicas, sociales y educativas; de éste último, nuestros estudiantes por varios factores han abandonado con la conexión de las clases virtuales, es nuestra preocupación urgente, en recuperar a los estudiantes que terminen el año escolar; no podemos hablar de calidad educativa, la igualdad, la equidad, e inclusión, sino hemos conseguido atender a su debido tiempo.

\section{Referente teórico}

La gestión educativa se enmarca en la gestión institucional y pedagógica, la existencia de la misma se da por los estudiantes y docentes, y éstas deben responder a la comunidad para mejorar el nivel de aprendizaje de acuerdo a los estándares y el dominio de las competencias en los ciclos, niveles y modalidades del sistema escolar. Una teoría concreta en la funcionalidad del sistema educativo, es la acción comunicativa entre los actores de las distintas entidades para enfrentar una situación; es bien sabido, el trabajo en equipo, renueva voluntades, desarrolla creatividad, da imaginación y por ende la innovación, bajo esas características hemos enfrentando, con ahínco en romper algunos viejos paradigmas de gestión pública; donde, se espera una solicitud para atender a la comunidad, nosotros nos hemos acercado a ellos para atender las dificultades en el sitio del problema con una comunicación asertiva.

En la gestión pedagógica los equipos de especialistas han involucrado, el aprendizaje colaborativo, movilizando a estudiantes de nivel superior, personalidades y autoridades públicas y privadas a fin de atender las necesidades más urgentes en la gestión educativa, especialmente en la conectividad digital, como lo establece el concepto de la internet en una red de interconexión propiamente sistémico; la práctica de la interdisciplinariedad como producto del bien en común para mejorar el servicio de la calidad educativa basado en la persona humana, el cual se refiere a los estudiantes como centros de interacción, según el modelo socioconstructivista.

El sistema de gestión institucional tiene muchas barreras en su funcionalidad, es importante afianzar a un cambio de las personas para un proceso de gestión educativa eficiente y eficaz; ya que, el actuar de toda autoridad educativa es viabilizar una oportuna atención con los recursos que cuenta, con un enfoque humanista, social y una administración participativa en el planeamiento de la enseñanza y el aprendizaje, bajo una dirección de liderazgo del equipo de los actores educativos en la región; los procesos estratégicos deben desarrollarse en el marco de la modernización de la gestión 
pública, tomando un sentido proactivo, participativo con una línea integradora desde el órgano superior a través de políticas que recojan experiencias exitosas de gestión a nivel local, nacional e internacional; lo que nos preocupa es que siendo una país diverso, se siga una línea administrativa vertical, soslayando toda forma de transversalidad en la gestión pública.

\section{Desarrollo de la experiencia}

La planificación estratégica en el sistema educativo del país, estaba prediseñado a mérito de ejecutar la campaña del "buen inicio del año escolar" del año 2020, se había hecho todo el trabajo en equipo, con todo el corporativo de las autoridades educativas, políticas y sociales a fin de lograr y acoger a los estudiantes de manera presencial. Nuestro punto de vista, es que enfrentamos una emergencia educativa, al cambiar nuestro enfoque, en el uso de los recursos digitales, nuestra infraestructura digital era ineficiente, había que hacer una mirada sistémica para resolver un problema socioeducativo, afectado gravemente por la pobreza, por la ausencia permanente del Estado en su dimensión integral, estamos frente a una problemática global; pero en la región, había que emprender mucho trabajo para articular las acciones de alianzas estratégicas y sobre todo comunicarse con instituciones, que también expresan su preocupación como tema prioritaria a la educación.

La articulación entre las instituciones, no, es solo teoría; también es práctico; nuestra experiencia ha demostrado enlazar una agenda, de cerrar brechas digitales, con una propuesta de atención prioritaria a la educación. Autoridades ediles se han sumado a implementar con la instalación de antenas para la captación de la señal electromagnética y mecánica a través de la web, televisión y radio para la implementación de la estrategia "aprendo en casa" (figura 01), y recién explicitar, nuestro verdadero problema de conectividad.

Figura 01. Aprendizaje remoto

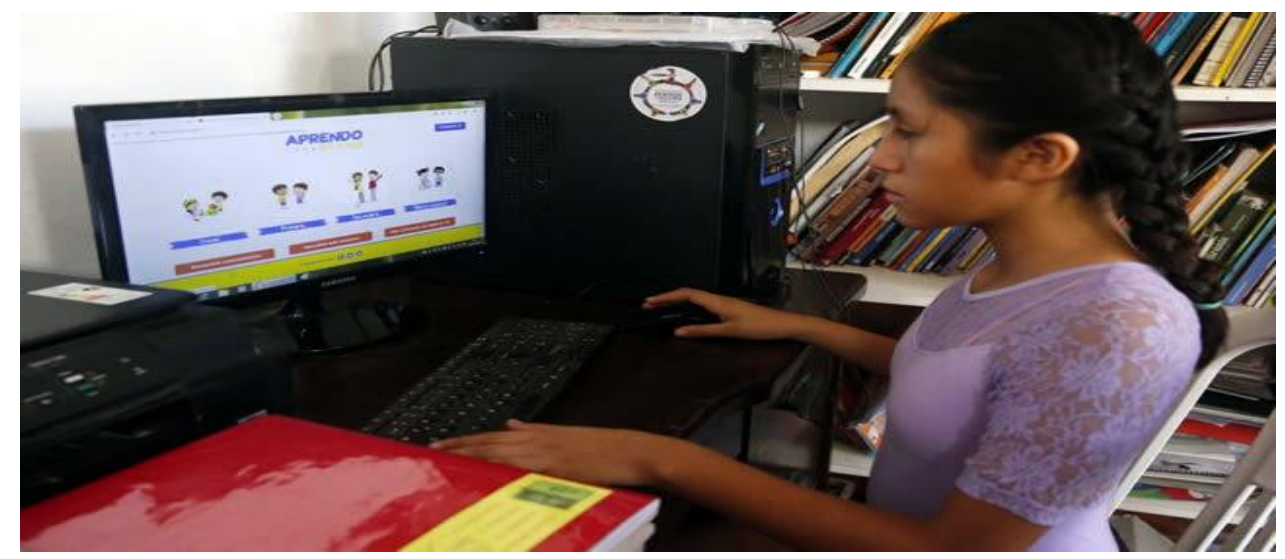

Fuente:https://www.drelm.gob.pe/drelm/wp-

content/uploads/2020/08/standard_acompanamientodocente1808.jpg

Ya estando en el centro de la pandemia, nuestro actuar estaba visionado en atender a los estudiantes de todos los niveles; pero en ese transcurrir, los niños, niñas y adolescentes, hasta jóvenes estudiantes, estaban en plena actividad física, caminando longitudes distantes, colgados de radios o simplemente buscando una señal electromagnética; había

Gestión educativa en tiempos de pandemia de la Región Puno, 2020 - Benavente, Mario DOI: https://doi.org/10.18800/cige2020.003 
cambiado nuestra imagen a "aprendo en cerro" (figura 02). Nuestro problema, fue lapidaria; que los usuarios más vulnerables estaban atendidos de manera deficiente.

Figura 01:

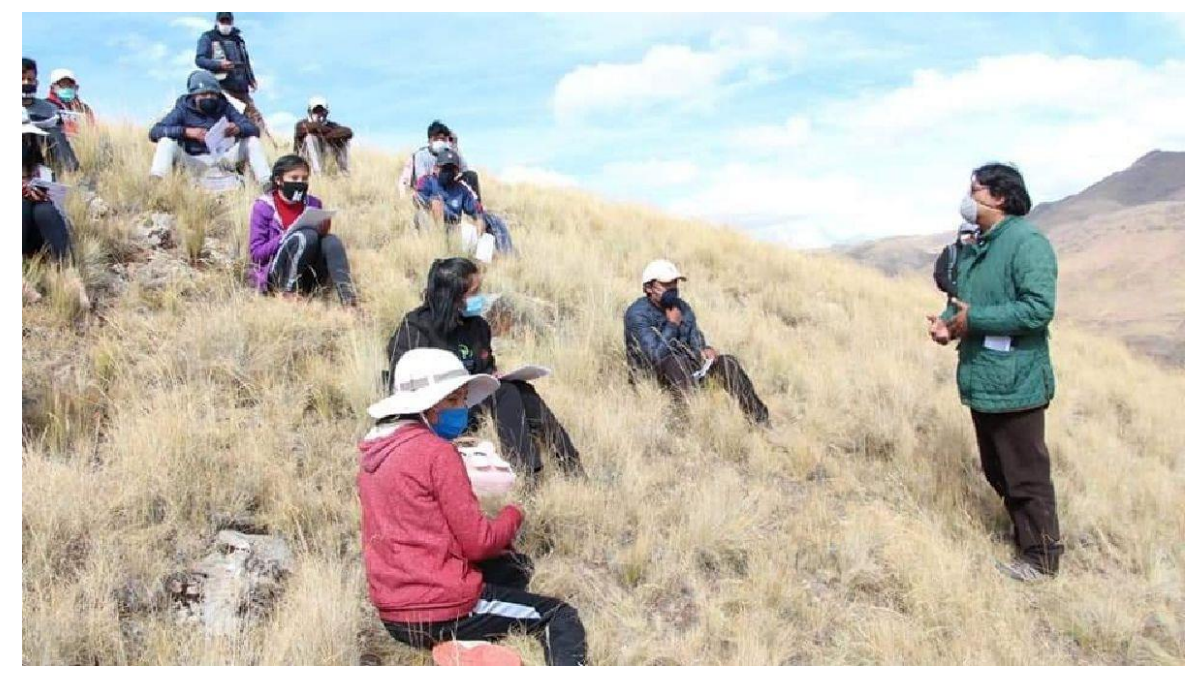

Fuente: https://e.rpp-noticias.io/xlarge/2020/06/18/345034_959148.jpg

Comprender ésta acción educativa, no parece, conforme se plantea los principios de la ley general de la educación; la equidad, la inclusión y la calidad, eran términos distantes a la realidad de la región u otras al interior del país. Pero lo estamos viviendo. Similar situación, estaban viviendo nuestros estudiantes en la zona de la selva de nuestro territorio en la región; nuestra tarea ha sido titánico en aras de atender la cobertura digital, se ha superado en algo, a través de instituciones públicas y privadas, y la iniciativa de la misma institución de poder entregar los chips de datos de telefonía celular, para reivindicar el derecho fundamental de un acceso a la calidad de educación.

La intervención conjunta, con planificación y mediación de los actores involucrados en el desarrollo de las competencias, ha motivado toda la estrategia de contextualización con herramientas de textos físicos a los estudiantes que no tienen conectividad; para los que egresan la educación secundaria, estamos atendiendo con guías de prospectos de admisión físicos en las áreas fundamentales, y la mediación a través de videoconferencias sincrónicas y asincrónicas, con ello queremos garantizar el nivel de aprendizaje a la totalidad de estudiantes en la gestión pública; para los maestros, se ha diseñado una estrategia de cursos en el uso de las herramientas de tecnología, para que haya un acercamiento a la realidad virtual e interactuar con los estudiantes; existe una movilización conjunta por los estudiantes y la educación; muchos autoridades, apenas han entendido la gravedad de la situación y se están encaminando en la estrategia de cerrar esa brecha existente, la desigualdad en el acceso a una educación de calidad.

\section{Resultados}

Las autoridades educativas hemos propuesto enfrentar la emergencia con trabajo en equipo, para ello se ha sincerado la cantidad de estudiantes que no tienen acceso a la conectividad y elaborado un mapeo para la intervención oportuna en la atención 
Congreso Internacional de Gestión Educativa, 06 y 07 de noviembre de 2020.

Pontificia Universidad Católica del Perú

prioritaria por ser un derecho fundamental. Las tantas actividades, se han concretizado en tareas que se emprende, en la búsqueda de alianzas estratégicas con instituciones y autoridades locales para la instalación de antenas y acceso a internet, éste ha sido con participación de muchas autoridades. En suma, los estudiantes que no tienen conectividad ya no emprenden largas caminatas para recibir su sesión de aprendizaje virtual.

\section{Conclusiones}

La gestión educativa está ligada en el liderazgo de comunicación, participación y la búsqueda de alianzas estratégicas con otras entidades del Estado, para que los estudiantes accedan a la estrategia "aprendo en casa" de manera oportuna y en las mismas condiciones por ser un derecho fundamental.

La mediación en la acción pedagógica, con asistencia técnica en el uso de las herramientas digitales hacía docentes y colaboradores ha sido de suma importancia y el seguimiento socioemocional en convenio con entidades que responden a la misma es un factor esencial para el aprendizaje de los escolares.

\section{Agradecimientos}

Agradezco a la PUCP por haberme hecho la invitación, para participar en tal certamen académico; asimismo agradezco a todo el equipo de trabajadores de la Dirección Regional de Educación Puno; asimismo a Dr. Teófilo Yucra Quispe, por su aporte en la sistematización, guía y redacción del artículo.

\section{Referencias bibliográficas}

Adelman, M. A., \& Lemos, R. (2019). Gestión para los aprendizajes. Repositorio institucional - MINEDU. http://repositorio.minedu.gob.pe/handle/MINEDU/6805

Alfaro, S., González, M. E., Mujica, L., Segato, R., \& Villasante, M. (2007). Educar en Ciudadanía Intercultural: Experiencias y retos en la formación de estudiantes universitarios indígenas. En Pontificia Universidad Católica del Perú. Pontificia Universidad Católica del Perú. Fondo Editorial. http://repositorio.pucp.edu.pe/index/handle/123456789/53738

Capacidades, P. M. de E. D. G. de G. D. D. de F. de. (2017). Sistematización de las buenas prácticas del I Concurso 20 en Gestión: Reconocimiento a las Buenas Prácticas de Gestión Educativa en las Unidades de Gestión Educativa Local 2016. Ministerio de Educación. http://repositorio.minedu.gob.pe/handle/MINEDU/6741

Educación, C. C. N. de, \& Trahtemberg Siederer, L. (2014). Gestión eficaz para fortalecer la Escuela Pública. http://repositorio.minedu.gob.pe/handle/123456789/3742

Gutiérrez Rubio, S. P., Heredia Morales, A. M., Horna Vásquez, E. R., \& Peña Banda, L. R. (2018). Planeamiento estratégico para la región Puno. Pontificia Universidad Católica del Perú. http://tesis.pucp.edu.pe/repositorio/handle/20.500.12404/12587 
Congreso Internacional de Gestión Educativa, 06 y 07 de noviembre de 2020.

Pontificia Universidad Católica del Perú

Peruana, F. F. N. de D. de la E. (2013). Guía de gestión de proyectos de innovación pedagógica. Proyectos Jugando Aprendo I y Jugando Aprendo II. Ministerio de Educación. http://repositorio.minedu.gob.pe/handle/123456789/4576

Proyecto Educativo Regional Concertado 2017- 2025. (s. f.). Recuperado 28 de septiembre de 2020, de http://www.drepuno.gob.pe/web/2011-11-14-19-2905/direccion/12-paginas/2126-proyecto-educativo-regional.html

Revilla Figueroa, D. (2007). La evaluación y autoevaluación institucional en las escuelas. En Pontificia Universidad Católica del Perú. Pontificia Universidad Católica del Perú. Fondo Editorial. http://repositorio.pucp.edu.pe/index/handle/123456789/166006

Samuel Gento Palacios. (2018). Gestión, dirección y supervisión de Instituciones y Programas de tratamiento educativo de la diversidad. http://archive.org/details/podcast_tratamiento-educativo-de-la-di_gestion-direcciony-supervisi_1000411443637 